Archives

$1 \mid 1988$

Varia

\title{
L'histoire sociale en France et en Allemagne fédérale : de l'ignorance cordiale aux promesses d'un nouveau dialogue
}

\section{Hartmut Kaelble}

Traducteur : Traduction et adaptation française : Étienne FRANÇOIS

\section{(2) OpenEdition Journals}

\section{Édition électronique}

URL : http://journals.openedition.org/ccrh/2969

DOI : $10.4000 /$ ccrh.2969

ISSN : $1760-7906$

\section{Éditeur}

Centre de recherches historiques - EHESS

\section{Édition imprimée}

Date de publication : 2 avril 1988

ISSN : 0990-9141

\section{Référence électronique}

Hartmut Kaelble, «L'histoire sociale en France et en Allemagne fédérale : de l'ignorance cordiale aux promesses d'un nouveau dialogue», Les Cahiers du Centre de Recherches Historiques [En ligne],

1 | 1988, mis en ligne le 13 avril 2009, consulté le 21 avril 2019. URL : http://journals.openedition.org/ ccrh/2969; DOI : 10.4000/ccrh.2969

Ce document a été généré automatiquement le 21 avril 2019.

Article L.111-1 du Code de la propriété intellectuelle. 


\title{
L'histoire sociale en France et en
}

\section{Allemagne fédérale: de l'ignorance cordiale aux promesses d'un nouveau dialogue}

\author{
Hartmut Kaelble \\ Traduction : Traduction et adaptation française : Étienne FRANÇOIS
}

1 Dans l'essor qu'a connu l'histoire sociale en Europe pendant les vingt dernières années, le Rhin a représenté une frontière plus impénétrable que la Manche - voire que l'Atlantique. Les spécialistes d'histoire sociale en Allemagne ont alors noué des contacts beaucoup plus étroits avec leurs collègues anglais ou américains qu'avec leurs collègues français, et la recherche allemande a reçu plus d'impulsions en provenance des États-Unis, mais aussi d'Angleterre, que de la France. D'autre part, il ne fait pas de doute que les spécialistes d'histoire sociale en France ont fait bien plus de cas de la recherche anglo-saxonne que de la recherche allemande. Les succès remportés aux États-Unis par l'École des Annales ont par ailleurs conféré aux relations entre historiens des deux pays une intensité telle qu'elles n'en n'avaient jamais connue. Les historiens français ont certes entendu parler de l'essor de l'histoire sociale en Allemagne fédérale - mais ils continuent d'en faire peu de cas.

2 Ce désintérêt réciproque de deux écoles d'histoire sociale aussi importantes l'une que l'autre ne laisse pas de surprendre. Du côté allemand en particulier, plusieurs historiens se sont interrogés sur les raisons de l'étonnant contraste entre la célébrité, voire la consécration de l'École des Annales et son absence à peu près complète d'influence sur l'histoire sociale de l'Allemagne fédérale. Pour rendre compte de cet état de fait, on avance communément six raisons principales :

$3 \quad \mathbf{1}$ - La différence la plus manifeste, sinon la plus importante, entre l'histoire sociale des Annales et l'histoire sociale allemande, serait d'abord due au fait qu'elles ne s'intéressent pas aux mêmes époques chronologiques. Effectivement, la grande majorité des études 
entreprises dans l'esprit des Annales en France - au même titre d'ailleurs que la plupart des débats de fond menés par les historiens français - portent sur l'époque moderne et la fin du Moyen Age. Les deux historiens français les plus connus à l'étranger, F. Braudel et E. Le Roy Ladurie, ont l'un comme l'autre avant tout travaillé sur cette période, négligeant relativement les $\mathrm{XIX}^{\mathrm{e}}$ et $\mathrm{xx}^{\mathrm{e}}$ siècles. Du côté allemand en revanche, la préférence manifeste va aux $\mathrm{XIX}^{\mathrm{e}}$ et $\mathrm{xx}^{\mathrm{e}}$ siècles, tandis que l'époque moderne fait en quelque sorte figure de parent pauvre (quelles que soient par ailleurs les qualités souvent éminentes des rares historiens qui s'y intéressent). La différence de thématique des spécialistes d'histoire sociale français et allemands, différence accentuée par des contrastes d'approches sur lesquels nous reviendrons, a donc eu pour effet de les rendre largement étrangers les uns aux autres, voire de transformer leurs échanges en véritables dialogues de sourds ${ }^{1}$.

Cette première explication du désintérêt réciproque des spécialistes d'histoire sociale français et allemands, a longtemps paru d'autant plus convaincante qu'elle semblait liée aux spécificités de l'histoire de chaque pays. La préférence accordée du côté français à l'époque moderne était ainsi mise en rapport avec une interrogation sur le pourquoi et le comment de l'événement le plus important de l'histoire de la France contemporaine, à savoir la Révolution; du côté allemand en revanche, l'intérêt porté à la période contemporaine était mis en rapport avec la volonté de comprendre les antécédents et les causes de la césure la plus décisive de l'histoire de l'Allemagne contemporaine, c'est-àdire le nazisme. Dans une telle perspective, loin d'être le fait du hasard, la divergence d'orientation des spécialistes d'histoire sociale des deux pays paraissait au contraire la conséquence logique et inévitable des contrastes d'évolution de l'histoire de leurs pays respectifs.

$5 \quad 2$ - La seconde raison mise en avant pour rendre compte des différences entre la recherche française et la recherche allemande tiendrait à une différence d'approche des liens entre histoire politique et histoire sociale. Nombre de spécialistes d'histoire sociale en Allemagne Fédérale ont en effet longtemps accordé plus d'importance que leurs collègues français à l'impact des événements, des structures et des décisions politiques sur l'histoire sociale, les prenant plus souvent comme point de départ de leur recherche. En Allemagne Fédérale bien plus qu'en France, l'histoire sociale a été ainsi perçue comme un moyen nouveau et plus moderne d'arriver à une meilleure compréhension et une explication plus satisfaisante du fait politique. Dans les années soixante, on voyait même dans ce qu'on appelait alors «l'histoire sociale politique » un progrès non seulement par rapport à la tradition historiographique allemande, mais aussi par rapport à l'École des Annales. A l'inverse, on insistait sur l'absence de dimension politique de la recherche française, sur sa propension à privilégier des champs de recherche non soumis à l'influence des décisions d'ordre politique (sinon marginalement), tels la démographie historique, l'histoire de la famille ou l'histoire des mentalités. Prenant rarement la politique pour point de départ de ses enquêtes, l'histoire sociale française, dans sa thématique comme dans ses méthodes, paraissait ainsi d'un faible recours aux historiens allemands soucieux d'«histoire sociale politique». De ce fait également, l'École des Annales paraissait bien plus étrangère aux historiens d'Allemagne Fédérale que les recherches d'histoire sociale effectuées en Angleterre ou aux États-Unis².

3 - La troisième différence - très étroitement liée à la première - a trait à la conception du temps et de la durée. Du côté français, les impulsions lancées par l'article-programme de Braudel sur la longue durée se sont révélées extrêmement fécondes; les enquêtes sur 
des structures sociales ou mentales de «longue durée » se sont multipliées, centrées sur l'étude d'aspects du passé aujourd'hui révolus ; cet intérêt porté à la longue durée et aux dimensions révolues du passé est au reste inséparable de la préférence donnée par la recherche française à l'étude du Moyen Age et de la période moderne, périodes plus éloignées de la nôtre et marquées par une plus grande permanence des structures.

7 A l'inverse, les spécialistes allemands d'histoire sociale se sont intéressés de préférence à l'étude des changements sociaux, des processus évolutifs et des transformations caractérisant un passé plus récent et liées le plus souvent à l'avènement de la démocratie moderne, à l'industrialisation et à leurs répercussions. Ils se sont par là même attachés à des phénomènes apparemment bien plus proches de la situation contemporaine et bien moins «étrangers" que les thèmes abordés par la recherche française. Tout en reconnaissant le côté brillant et souvent même extraordinairement impressionnant de la recherche historique française, les spécialistes allemands d'histoire sociale ne pouvaient s'empêcher de déplorer son manque d'intérêt pour l'étude du changement politique et social des $\mathrm{XIX}^{\mathrm{e}}$ et $\mathrm{XX}$ e siècles, la jugeant de ce fait peu utile pour eux ${ }^{3}$.

$8 \quad 4$ - Ces différences déterminèrent à leur tour un contraste essentiel dans l'attitude des spécialistes d'histoire sociale français et allemands envers les théories. Du côté des Annales, l'étude et l'approfondissement des théories élaborées par les autres sciences de l'homme et de la société - en vue de leur utilisation ultérieure dans la recherche historique - n'auraient pas constitué un objectif prioritaire. Certes, les historiens de l'École des Annales se sont toujours efforcés de travailler dans l'interdisciplinarité, ils se sont toujours montrés ouverts aux problématiques des autres disciplines et les ont reprises à leur compte. Il n'en reste pas moins que la réflexion et la discussion théorique n'ont pas occupé une place de premier plan dans le programme de l'École des Annales. A l'inverse, les spécialistes allemands d'histoire sociale en ont fait une de leurs priorités essentielles ; moyen privilégié de marquer leurs distances par rapport à l'historiographie traditionnelle, le recours à la théorie a été posé par eux comme un élément constitutif d'une histoire sociale nouvelle et véritablement scientifique. Qu'en ce domaine les réalisations effectives soient restées en-deçà des intentions affichées et des proclamations de principe ne saurait certes être contesté. Il n'en reste pas moins que depuis les années soixante, les spécialistes d'histoire sociale allemands ont assurément accordé aux questions théoriques et aux problèmes posés par leur mise en œuvre une attention sans précédent dans la tradition historiographique allemande. Or, en ce domaine aussi, ils ne trouvaient guère d'équivalents ni de références du côté de l'École des Annales, comme si, là encore, un fossé presque infranchissable séparait l'histoire sociale faite en France de l'histoire sociale faite en Allemagne Fédérale'.

95 - On a également proposé d'expliquer les différences entre la tradition des Annales et la nouvelle histoire sociale allemande par les différences de contexte socio-politique ayant accompagné leur naissance. On a fait ainsi remarquer que la relative stabilité démocratique de la France d'entre les deux guerres avait favorisé l'émergence de l'histoire sociale. Même si ces tendances étaient au départ minoritaires, elles ne furent jamais gravement menacées - pas même pendant la période de l'occupation nazie : n'estce pas précisément durant sa captivité en Allemagne que $\mathrm{F}$. Braudel rédigea la "Méditerranée ", le maître-livre des Annales? En France également, le marxisme n'a jamais fait l'objet d'un refus absolu ni n'a été transformé en tabou, et le rôle essentiel joué dans la Résistance par le parti communiste lui a même donné une importance accrue. La première moitié $\mathrm{du} \mathrm{xx}^{\mathrm{e}}$ siècles aurait ainsi donné aux différents courants de l'histoire 
sociale en France des conditions de développement favorables ; par la suite, les historiens français auraient su, même dans l'adversité, en faire le meilleur usage, élaborant un programme de recherche et des perspectives d'avenir qu'ils allaient peu après mettre en œuvre et concrétiser à la faveur de l'explosion économique et universitaire de l'aprèsguerre. La VIe section de l'École des Hautes Études (future E.H.E.S.S.) ainsi que la Maison des Sciences de l'Homme sont le résultat le plus visible de cette expansion réussie; sans exemples dans le reste de l'Europe par leurs dimensions, ces institutions sont l'une des raisons des succès de l'École des Annales.

10 Rien de comparable du côté allemand. Obsédée après 1918 par le problème de la « responsabilité » allemande dans le déclenchement de la guerre, la recherche historique allemande pendant la République de Weimar néglige l'histoire sociale. Les conséquences négatives de cette évolution sont considérablement aggravées par le nazisme et l'étouffement de la liberté intellectuelle, l'isolement des historiens choisissant le chemin de l'« exil intérieur » et la nazification de certains domaines de l'histoire sociale (histoire rurale, démographie historique). Dans ces conditions, le redémarrage de l'histoire sociale après guerre se fait à bien des égards à partir de zéro, dans des conditions radicalement différentes de celles de la France.

116 - Dernier point enfin : certaines évolutions récentes semblent aller dans le sens d'une aggravation des différences entre la recherche française et la recherche allemande. A s'en tenir aux apparences, l'histoire sociale en Allemagne Fédérale vient d'être agitée par un nouveau conflit, le conflit entre, d'un côté, le courant de «l'histoire comme science sociale ", courant plus ancien, très porté sur la théorie, donnant la préférence à l'étude des structures, parfois féru de quantification, soucieux de conclusions de portée générale et attachant une grande importance au rôle de l'État, et, de l'autre, le courant plus récent de l'Alltagsgeschichte (histoire du quotidien), plus réservé à l'égard des théories, résolument "micro-historique», s'intéressant par priorité à l'expérience vécue et aux histoires de vies et souvent tenté par un populisme dépolitisé. Or ce conflit, aux manifestations parfois fort vives, n'a pas d'équivalent en France. On peut bien sûr se demander si l'essor récent de l'Alltagsgeschichte en Allemagne fédérale n'est pas la marque d'une influence accrue de l'esprit des Annales: quand elle tente de préciser les fondements théoriques et méthodologiques, l'Alltagsgeschichte fait effectivement très souvent référence aux travaux récents d'histoire sociale et d'histoire anthropologique parus en France, si bien qu'à la limite, on pourrait presque présenter le conflit entre ces deux courants de l'histoire sociale allemande comme un conflit entre une conception de l'histoire sociale spécifiquement allemande et une conception de l'histoire sociale puisant son inspiration dans les Annales. Quoi qu'il en soit, ce conflit interne à l'histoire sociale allemande ne rencontre du côté français ni analogies, ni compréhension ${ }^{5}$. Ainsi semblerait se confirmer l'idée selon laquelle la recherche française et la recherche allemande en histoire sociale continueraient de progresser sur des voies étrangères l'une à l'autre et n'auraient finalement pas grand-chose à se dire.

Ces explications sont loin d'être dépourvues de pertinence et on ne saurait leur reprocher de tourner le dos à la réalité. Elles ont été avancées par des historiens allemands connaissant bien la recherche française. Il s'agit là incontestablement de jugements fondés et non de préjugés. Mais alors, une question se pose : faut-il vraiment que nous ayons sur le continent deux pôles de recherche en histoire sociale aux horizons intellectuels radicalement différents, deux pôles qui coexistent sans se rencontrer, qui, tout en se respectant, s'ignorent aimablement, quand ils n'entrent pas en concurrence 
l'un avec l'autre dans les autres pays européens ou aux États-Unis ? Faut-il aller jusqu'à penser que le fossé entre spécialistes d'histoire sociale en France et en Allemagne Fédérale est resté aussi infranchissable et que les multiples colloques franco-allemands, la création d'un Institut Historique Allemand à Paris et d'une Mission Historique Française à Göttingen, les accords existant entre les Universités de Bielefeld, de Berlin et l'École des Hautes Études, les programmes d'échanges de la Maison des Sciences de l'Homme et du CNRS, du DAAD, de la Fondation Volkswagen et de la DFG, les très nombreux contacts, souvent amicaux, entre historiens des deux pays, n'ont rien changé à cet état de fait? L'ancienne confrontation des "ennemis héréditaires " n'aurait-elle fait place qu'à une indifférence cordiale masquant à peine la persistance d'un profond fossé entre deux univers culturels étrangers l'un à l'autre ? Va-t-on voir se prolonger à l'avenir l'absence de participation sérieuse des historiens français aux débats de fond de l'histoire sociale allemande et inversement l'absence de participation des historiens allemands aux débats de l'histoire sociale française et plus généralement européenne - à l'inverse de ce à quoi les Allemands ont habitué, pour leur plus grand profit, les historiens anglo-saxons? A l'heure actuelle, la réponse à cette question ne peut être que négative. Il n'existe pas de livre écrit par un Français sur l'histoire sociale de l'Allemagne dont la portée puisse se comparer à celle des études de D. Blackbourn, R. Evans, G. Feldman, P. Gay, V. Lidtke, C. Maier, T. Mason, A. Mayer, B. Moore, F. Ringer, J. Sheehan, H.A. Turner - pour ne citer que les noms les plus connus. La seule exception est la thèse d'État de Pierre Ayçoberry ${ }^{6}$. Inversement, il n'existe pas d'étude sur l'histoire sociale de la France écrites par un Allemand dont la portée soit comparable à celle des études de R.Cobb, R. Forster, S. Kaplan, J. Scott, W. Sewell, A. Sutcliffe, J. Merriman, C. Tilly, E. Weber ou T. Zeldin - là encore, il s'agit d'une énumération non limitative. Ni en France, ni en Allemagne Fédérale d'ailleurs, n'existent d'associations regroupant l'ensemble des historiens travaillant sur l'histoire de l'autre pays, alors qu'il existe par exemple en RFA des associations de spécialistes de l'histoire de l'Amérique du Nord, de l'Angleterre ou de l'Italie.

13 En dépit de la négativité de ce premier constat, le pessimisme n'est cependant pas de mise. Car dans un pays comme dans l'autre, l'essor de l'histoire sociale s'est accompagné d'importantes mutations intérieures - mutations dont on n'a pas encore assez pris la mesure, mais qui ont eu pour effet de faire reculer sensiblement ce qui jusque là séparait la recherche française de la recherche allemande. Un historien allemand arrivant en France, il y a vingt ans, était alors avant tout frappé par la différence des approches et des problématiques ; aujourd'hui, au contraire, il est surpris par la proximité des thèmes de recherche et des méthodes, si bien qu'en réalité, l'opinion communément admise de l'absence de points communs entre l'École des Annales et l'histoire sociale d'Allemagne Fédérale correspond de moins en moins à la réalité. Ce rapprochement réciproque est à bien des égards plus avancé qu'il n'y parait ; il tient beaucoup plus à des évolutions d'ordre interne qu'au renforcement de l'influence exercée par l'école historique d'un pays sur l'autre - ce qui le rend à la fois plus essentiel et plus prometteur pour l'avenir.

$14 \mathbf{1}$ - Les historiens allemands n'ont pas encore vraiment pris conscience de la disparition des anciens contrastes de priorité chronologique. Il y a maintenant en France au moins autant d'excellents spécialistes d'histoire sociale du XIX ${ }^{\mathrm{e}}$, sinon $\mathrm{du} \mathrm{xx}^{\mathrm{e}}$ siècles, qu'en Allemagne Fédérale; quatre créations récentes témoignent de cette reconversion de l'histoire sociale en direction de l'époque contemporaine : celle de l'Institut d'Histoire du Temps Présent dirigé par F. Bédarida, celle du Centre de Recherche d'Histoire des Mouvements Sociaux dirigé par A. Prost, celle de l'Institut d'Histoire Moderne et 
Contemporaine dirigé par F. Caron, celle enfin du Centre d'Histoire Économique et Sociale de la Région Lyonnaise dirigé par M. Garden et Y. Lequin. Tous ces instituts sont dirigés par des spécialistes de l'histoire sociale des $\mathrm{XIX}^{\mathrm{e}}$ et $\mathrm{xx}^{\mathrm{e}}$ siècles et font dans leurs programmes de recherche une place essentielle à l'histoire contemporaine. À l'École des Hautes Études en Sciences Sociales, le Centre de Recherches Historiques, sous l'impulsion de R. Chartier, et maintenant de L. Bergeron, pratique une politique décidée d'ouverture en direction de l'époque contemporaine (cf. la nomination récente de deux spécialistes du $\mathrm{XIX}^{\mathrm{e}}$ siècle, L. Bergeron comme directeur et P. Bourdelais comme directeur-adjoint du Centre). Du côté des revues, la tendance est la même, qu'il s'agisse de l'importance croissante prise par le Mouvement Social (qui fait actuellement figure en France de pendant de la revue Geschichte und gesellschaft en RFA), ou de la création récente de la revue Vingtième Siècle, faisant elle aussi une large place aux travaux d'histoire sociale. Les Annales elles-mêmes n'échappent pas à cette réorientation, si l'on en juge par la multiplication, au cours des dix dernières années, des articles d'histoire contemporaine parus dans cette revue. Loin d'être l'effet d'une décision autoritaire prise d'en haut, cette réorientation en cours correspond au contraire à l'émergence en France d'une nouvelle génération d'historiens travaillant sur l'histoire sociale des $\mathrm{xIX}^{\mathrm{e}}$ et $\mathrm{xx}^{\mathrm{e}}$ siècles, tels M. Agulhon, P. Ayçoberry, L. Bergeron, S. Chassagne, A. Corbin, A. Daumard, J. Dupâquier, E. François, P. Fridenson, J.P. Goubert, R. Hubscher, Y. Lequin, M. Perrot, A. Prost, D. Woronoff - pour ne citer que les plus influents d'entre eux. Il n'est plus possible aujourd'hui d'affirmer - comme on pouvait le faire il y a une dizaine d'années - que la recherche en histoire sociale sur l'époque contemporaine est plus active et plus productive en Allemagne Fédérale qu'en France. La seule différence entre les deux pays tient au fait qu'en France, en raison du prestige et de l'antériorité des recherches en histoire moderne, ces recherches nouvelles consacrées à l'histoire sociale de la France contemporaine ont encore une certaine difficulté à « percer ».

2 - La montée en puissance de l'histoire sociale de la France contemporaine s'est accompagnée d'un changement dans l'approche du fait politique, entrânant là aussi un rapprochement avec l'Allemagne Fédérale, si bien qu'on ne peut plus aujourd'hui opposer d'un côté une histoire sociale " politique " à l'allemande et de l'autre une histoire sociale «apolitique» à la française. Bien souvent, en effet, c'est le désir d'arriver à une explication plus en profondeur de l'histoire politique des $\mathrm{XIX}^{\mathrm{e}}$ et $\mathrm{xx}^{\mathrm{e}}$ siècles, qui est à l'origine des nouvelles enquêtes sur l'histoire sociale de la France contemporaine: les études d'Y. Lequin sur les ouvriers de la région lyonnaise au XIX ${ }^{e}$ siècle, de M. Perrot sur les ouvriers en grève dans la France du XIX ${ }^{e}$ siècles, d'A. Prost sur les Anciens Combattants entre les deux guerres, de M. Agulhon sur la sociabilité et la culture politique des XIX et $\mathrm{xx}^{\mathrm{e}}$ siècles, d'A. Corbin sur l'archaïsme du Limousin au $\mathrm{XIX}^{\mathrm{e}}$ siècle, sont à cet égard exemplaires. En profondeur, ces enquêtes ne sont pas sans rappeler les enquêtes lancées en Allemagne dans les années 1960 et 1970, à l'âge d'or de l'« histoire sociale politique ». La ressemblance n'est certes pas parfaite - ne serait-ce qu'en raison des contrastes de l'évolution politique des deux pays. L'essor du mouvement ouvrier dans la France d'avant 1914 n'est pas comparable à celui de l'Allemagne - et surtout il n'y a pas pris les mêmes formes institutionnelles ; la France n'a pas connu de révolution en 1918-1919; elle n'a pas été ravagée par l'inflation, les conséquences de la crise économique y furent moins dramatiques qu'en Allemagne; surtout elle n'a connu ni nazisme, ni coupure radicale en 1945. Cela explique que les historiens français donnent la préférence à d'autres thèmes que leurs collègues allemands, qu'à côté de l'histoire ouvrière, ils s'intéressent à des 
thèmes moins familiers aux historiens allemands, tels que la postérité de la Révolution, l'histoire de l'urbanisation, l'histoire du monde rural, ou celle de la vie privée. Mais fondamentalement, les approches sont les mêmes - ce qui rend le dialogue possible et fécond.

Mais si historiens français et historiens allemands se sont sensiblement rapprochés, cela tient aussi à l'évolution de la recherche historique allemande. A l'heure actuelle, l'histoire sociale en RFA n'est plus un simple appendice de l'histoire politique. Elle s'est diversifiée, perfectionnée, perdant ainsi progressivement les traits qui la singularisaient à ses débuts et se rapprochant d'autant d'une sorte de moyenne européenne. Au départ simple " substrat » de l'histoire politique, discipline auxiliaire pratiquée sans excessive rigueur et en quelque sorte de seconde main, l'histoire sociale s'est progressivement et sans bruit affirmée comme une discipline autonome et à part entière. Trois raisons rendent compte de cette évolution.

La première tient à la sophistication rapide des méthodes de l'histoire sociale, évolution due elle-même à la hausse très sensible des exigences qualitatives de la recherche. C'est ainsi que des enquêtes qui, à l'origine, se voulaient socio-politiques, sont finalement devenues des enquêtes purement sociales - par ailleurs souvent excellentes. Ceci a été le cas en particulier de plusieurs études portant sur le mouvement ouvrier et le milieu des employés. Or une telle évolution n'a rien de proprement allemand, puisqu'on l'observe de la même manière aussi bien en Angleterre qu'en France : l'enquête d'Y. Lequin sur les ouvriers de la région lyonnaise au $\mathrm{XIX}^{\mathrm{e}}$ siècle ou celle de G. Crossick sur l'aristocratie ouvrière londonienne sont toutes deux des exemples d'études initialement prévues pour servir de support à une problématique politique, mais qui, poussées par la logique de leur développement interne, sont devenues par la suite des enquêtes d'histoire sociale au sens fort du terme.

La seconde raison tient au changement fréquent de priorités effectué par les spécialistes d'histoire sociale en Allemagne. Sans remettre en question les liens entre histoire sociale et histoire politique, ces derniers n'en ont pas moins porté une attention croissante à l'étude des faits de civilisation matérielle et de mentalité, dans une conception élargie du fait politique. Dans cet état d'esprit, ils s'attachent davantage à l'étude des conditions d'existence des milieux sociaux et des classes sociales qu'à celle de leur degré de politisation; ils font passer l'étude des comportements quotidiens et des mentalités collectives avant celle des idéologies politiques, l'étude de la vie familiale avant celle de la politique de la famille, l'étude des conditions réelles d'existence des malades ou des personnes âgées avant celle de la politique sociale, l'étude des chances concrètes d'éducation avant celle des politiques éducatives, l'étude des conditions de vie concrètes pendant le nazisme avant celle des décisions politiques prises au sommet de la hiérarchie. Cet élargissement du regard historique et la place croissante faite à l'observation de la qualité de la vie dans l'étude du passé sont ainsi des évolutions qui ont contribué à rapprocher l'histoire sociale allemande de l'histoire sociale française.

19 La troisième raison vient de ce que, depuis un certain temps déjà, plusieurs historiens allemands ont pris pour sujet d'étude des thèmes très proches de ceux de leurs collègues français et dans lesquels la politique au sens classique du terme n'intervient que marginalement. Il en va ainsi par exemple des enquêtes sur l'histoire de la croissance urbaine, de celles sur les migrations ou la mobilité sociale, de celles sur l'histoire des domestiques, des gens de mer, des artisans ruraux, mais aussi des enquêtes sur l'enfance ou sur la vieillesse. On se tromperait à interpréter les développements de ces enquêtes 
comme la marque d'un détachement de la politique. Bien plus souvent, en réalité, ces enquêtes se proposent de préciser et de mesurer les limites des décisions et des interventions politiques; leur objectif est de retrouver les domaines de la réalité sociale qui opposent une résistance obstinée à l'entreprise de la politique et qui obéissent à d'autres règles d'évolution.

Ainsi peut-on constater que la place de la politique dans l'histoire sociale s'est redéfinie de part et d'autre du Rhin. Du côté français, la nouvelle génération lui accorde une place plus importante que celle qu'elle avait dans l'histoire sociale classique centrée sur le Moyen Age ou l'époque moderne. A l'inverse, la jeune génération en Allemagne a une approche beaucoup plus ouverte et diversifiée des rapports entre histoire sociale et histoire politique; dans un pays comme dans l'autre, on s'intéresse aujourd'hui davantage, dans une vue plus réaliste des choses, aux frontières de la politique.

213 - Dans l'histoire sociale telle qu'on la pratique actuellement en Allemagne Fédérale, le recours et la référence aux théories sont bien différents de ce qu'ils étaient il y a une vingtaine d'années. L'exigence théorique d'alors était en quelque sorte le mot d'ordre servant à regrouper tout un ensemble d'aspirations au changement. Ce mot d'ordre correspondait certes à une volonté d'élargissement et de précision des problématiques historiques grâce à l'aide des autres sciences humaines. Mais plus profondément, il était l'expression de la volonté des jeunes historiens allemands de "déprovincialiser » leur discipline, de l'arracher au retard qu'elle avait accumulé pour lui faire rejoindre la recherche américaine et européenne et la hisser à leur niveaux. Ce mot d'ordre était enfin l'expression d'une volonté de redéfinition de la finalité sociale de l'histoire comme discipline scientifique: après des décennies pendant lesquelles l'histoire avait été en Allemagne presque complètement absorbée par ses tâches de justification nationale, l'objectif était de lui assigner une nouvelle fonction « critique », d'en faire une science qui dresserait un bilan objectif de l'évolution économique, sociale et politique de l'Allemagne contemporaine et contribuerait ainsi à poser les fondements scientifiques de l'édification de l'Allemagne de l'avenir, d'une société allemande enfin exorcisée de ses démons, moderne et démocrate.

Une partie des objectifs que s'étaient fixés les rénovateurs de la recherche historique allemande il y a une vingtaine d'années sont entretemps devenus réalité. L'histoire sociale de l'Allemagne Fédérale peut aujourd'hui se mesurer d'égale à égale avec la production des écoles française, anglo-saxonne ou suédoise. Dans l'ensemble également, on peut considérer que l'ouverture de la recherche en histoire sociale sur les autres sciences humaines a été couronnée de succès. La redéfinition des finalités sociales de la discipline est enfin largement acquise. En revanche, l'optimisme planificateur des années soixante a fait place à un scepticisme généralisé et plus personne aujourd'hui n'ose encore prétendre que la recherche historique puisse aider à édifier un avenir meilleur. On attend plutôt qu'elle aide, soit au niveau national, soit au niveau local, à la redécouverte des identités collectives; or, pour répondre à cette nouvelle fonction sociale, l'exigence théorique ne présente plus la même nécessité. Un certain dégrisement a enfin succédé à l'enthousiasme théorique des années soixante : on s'est ainsi rendu compte des difficultés présentées par la mise en œuvre concrète de théories élaborées par d'autres disciplines, d'où à terme - sans pour autant remettre en cause l'essentiel, c'est-à-dire le souci d'une histoire ouverte, exigeante et pluridisciplinaire - un allégement progressif des pesanteurs théoriques des débuts et une ressemblance croissante entre la pratique des historiens allemands et celle de leurs collègues français. Non que de part et d'autre du Rhin les 
différences aient été totalement abolies, mais elles tiennent aujourd'hui davantage à des différences d'expression et d'écriture qu'à des divergences de principe.

4 - Par-delà ces rapprochements, il faut enfin tenir compte des débats et des évolutions qui ont affecté la communauté des spécialistes d'histoire sociale à l'échelle européenne. Depuis un quart de siècle, l'école française comme l'école allemande ont connu les mêmes grands courants; dans un pays comme dans l'autre, hier comme aujourd'hui, les principaux thèmes de discussion sont comparables, qu'il s'agisse de l'histoire de la classe ouvrière, de celle des grèves, de celle des minorités ouvrières avant 1914, de celle des entreprises ou des entrepreneurs, de celle de la mobilité sociale, des femmes, de la famille, ou, plus récemment, de celle de la bourgeoisie. Depuis un bon quart de siècle, les expériences et les débats de l'histoire sociale en France et en Allemagne Fédérale sont semblables; par voie de conséquence, les discussions autour des nouveaux thèmes de recherche se déroulent dans un pays comme dans l'autre sur un même horizon intellectuel, soit une situation profondément différente de celle qui caractérisait les années soixante.

Quelles seront les conséquences de ce rapprochement sur l'histoire sociale des deux pays? Va-t-on voir se multiplier les thèmes de recherche communs, voire les enquêtes communes? Peut-on enfin espérer un renforcement des influences réciproques? Les échanges entre Français et Allemands vont-ils enfin devenir aussi étroits que les échanges avec les historiens anglo-saxons ? Ou tout restera-t-il au contraire dans l'état antérieur? Continuera-t-on, à l'exception de quelques isolés assez téméraires pour s'intéresser à l'histoire du pays voisin, voire à y enseigner, à se laisser majoritairement rebuter par les obstacles linguistiques, les différences de style et d'expression, le cloisonnement des réseaux de communication et de discussion?

On se tromperait à coup sûr en plaçant trop haut la barre des attentes. D'un certain point de vue en effet, les rapports entre spécialistes d'histoire sociale français et allemands correspondent à la norme européenne. Les historiens allemands n'entretiennent pas de rapports plus étroits avec leurs collègues suédois, danois, hollandais ou italiens. Quant aux historiens français, ils n'ont pas, eux non plus, de rapports plus étroits avec leurs collègues espagnols, italiens ou belges. Entre les spécialistes d'histoire sociale des différents pays européens, la règle semble bien être celle du désintérêt cordial et partout ceux qui travaillent sur l'histoire d'un autre pays que le leur sont très minoritaires.

Cette constatation résignée ne doit cependant pas conduire au découragement. Les raisons ne manquent pas qui plaident en faveur d'efforts renouvelés pour venir à bout des isolements nationaux. Quelles que soient les réserves que l'on peut avoir à l'égard de projets trop ambitieux, il n'en reste pas moins que deux écoles d'histoire sociale aussi importantes sur le continent que l'école française et celle d'Allemagne Fédérale ont beaucoup à s'apporter et beaucoup à s'apprendre mutuellement, et que leur rapprochement ne peut que profiter à l'ensemble de la communauté historienne européenne. Jamais probablement les conditions n'ont été aussi favorables, ce qui doit inciter à savoir faire preuve d'autant d'imagination que de réalisme.

Dans une telle perspective, trois priorités s'imposent. La première consiste à développer les possibilités d'échange et de séjour dans le pays voisin. A côté des possibilités déjà offertes par diverses institutions - ainsi pour les Allemands les bourses de l'Institut Historique Allemand, les bourses du DAAD ou encore le Centre Européen de Rencontres en voie de constitution à Paris -, il conviendrait en particulier d'inventer des formules permettant des séjours de recherche et d'information de longue durée (six mois à un an); 
ces séjours devraient être libres de toute contrainte d'enseignement et assez longs pour permettre de surmonter des blocages psycho-linguistiques et garantir une réelle immersion dans le climat intellectuel et scientifique du pays voisin. Ils devraient être proposés aussi bien aux jeunes chercheurs qu'aux historiens confirmés et aux universitaires déjà en place.

La seconde priorité consiste à multiplier les possibilités de contact, de rencontre et d'échanges. Car si les contacts individuels ne manquent pas, les rencontres organisées, régulières et collectives font encore trop souvent défaut, à l'exception de quelques secteurs spécialisés (histoire des femmes, histoire orale, par exemple). Deux possibilités à cet égard sont à envisager. La première serait la constitution à l'intérieur de chaque pays de structures de rencontre et d'échange permettant aux spécialistes de l'histoire sociale de l'histoire du pays voisin de mieux se connaitre et d'avoir entre eux des contacts réguliers. La seconde serait l'organisation de rencontres régulières de spécialistes des XIX et $\mathrm{xx}^{\mathrm{e}}$ siècles en France et en Allemagne. Ces rencontres seraient centrées sur des thèmes de recherche étudiés dans un pays comme dans l'autre; loin de se limiter au petit groupe des spécialistes de l'autre pays, elles devraient au contraire assurer la rencontre d'historiens travaillant dans un pays comme dans l'autre sur des thèmes apparentés mais n'ayant pas eu jusque là la possibilité de se rencontrer. Elles devraient être ouvertes aussi bien aux historiens déjà arrivés qu'aux jeunes chercheurs. Elles devraient enfin savoir garder des dimensions restreintes, adopter la forme de réunions de travail de deux jours et tout faire pour favoriser la connaissance mutuelle dans un cadre aussi peu académique que possible. Une première rencontre de ce style a eu lieu en avril 1987 à Lyon, sur $l^{\prime}$ histoire sociale des entreprises aux $\mathrm{XIX}^{\mathrm{e}}$ et $\mathrm{xx}^{\mathrm{e}}$ siècles. Deux autres rencontres sur la bourgeoisie et sur la politique sociale sont prévues pour octobre 1988 et pour $1989^{7}$.

La troisième priorité a trait enfin à l'amélioration de l'information réciproque. La multiplicité des revues, l'obstacle de la langue, la prolifération de la « littérature grise » représentent autant d'obstacles à une circulation rapide de l'information. Pour lutter contre les inconvénients de cet état de fait, plusieurs possibilités sont à envisager. La première consiste à encourager les traductions; de divers côtés on s'y emploie, mais il s'agit là d'une modalité onéreuse et ne pouvant concerner au mieux que quelques ouvrages par an. La seconde consisterait à publier assez régulièrement des bulletins d'information bibliographiques et critiques faisant le point sur tel ou tel aspect de la recherche dans le pays voisin; très utiles (même s'ils se périment rapidement), de tels bulletins cependant ne s'improvisent pas et ne peuvent être réalisés que par des institutions bien implantées dans l'autre pays et équipées en conséquence, c'est-à-dire en fait par l'Institut Historique Allemand et la Mission Historique Française. Pourquoi enfin ne demanderait-on pas aux Instituts Français en Allemagne et aux Instituts Goethe en France de faire parvenir régulièrement aux historiens qui le souhaitent la table des matières des principales revues d'histoire sociale et, le cas échéant, les copies des articles qui les intéressent plus directement ? Cette formule est déjà mise en œuvre en Allemagne Fédérale par les centres culturels américains et donne à peu de frais les meilleurs résultats.

Il faut se rendre à l'évidence : l'histoire sociale en France et en République Fédérale ne sont plus aujourd'hui ce qu'elles étaient il y a vingt ans. Sans toujours s'en rendre compte, les chercheurs des deux pays se sont très sensiblement rapprochés dans leurs centres d'intérêt, leurs problématiques et leurs méthodes. Il y a vingt ans, la différence entre l'histoire sociale faite en France et celle faite en Allemagne Fédérale était une 
différence de nature ; elle n'est plus aujourd'hui qu'une question de nuances. Il est donc plus urgent que jamais de savoir dépasser les cloisonnements nationaux et les ignorances réciproques. L'organisation de rencontres régulières, une meilleure définition des programmes de séjour à l'étranger et une amélioration de la diffusion de l'information seraient certainement des étapes essentielles dans cette direction. Historiens français et historiens allemands ont autant à apporter les uns aux autres qu'à apprendre les uns des autres; leur rencontre ne pourra que profiter à l'histoire sociale de l'Europe tout entière.

\section{NOTES}

1. Voir par exemple: D. Groh, «Strukturgeschichte als «totale» Geschichte», dans : Vierteljahrschrift für Sozial-und Wirtschaftsgeschichte 58. 1971, pp. 317-319; M. Erbe, Zur neueren französischen Sozialgeschichtsforschung, Darmstadt, 1979, p. 21. Je remercie Etienne François d'avoir fort bien traduit cet article. Je remercie aussi Patrick Fridenson, Jürgen Kocka et Wolfgang Mager d'avoir fait des propositions importantes.

2. Voir K.E. Born, Neue Wege der Wirtschafts - und Sozialgeschichte in Frankreich: die Historikergruppe der "Annales", dans Saeculum, n ${ }^{\circ} 15.1964$, pp. 301-302, 307 ; Erbe, Sozialgeschichtsforschung, pp. 136-137: M. Erbe, Historisch-anthropologische Fragestellungen der Annales-Schule, dans: Historische Anthropologie, dirigé par H. Süssmuth, Göttingen, 1984, p. 26 ; H. Lutz, Braudel, «La méditerranée ». Zur Problematik eines Modellspruchs, dans : Schrift und Materie der Geschichte, dirigé par C. Honegger, Francfort, 1977, p. 35 ; voir aussi Th. Schieder, Strukturen und Persönlichkeiten in der Geschichte, dans: Historische Zeitschrift, 195, 1962, pp. 266, 273-275, 293

3. Voir Schieder, Strukturen, pp. 266, 273-275 ; J. Kocka, Sozialgeschichte, 2ème édit. Göttingen, 1986, p. 90 ; Groh, Strukturgeschichte, pp. 317-319; Born, Neue Wege, pp. 302-303.

4. Voir Born, Neue Wege, p. 307 ; Kocka, Sozialgeschichte, pp. 91-92, 108 : Honegger, Annales, p. 37.

5. Voir comme des échos français à ce conflit : E. François, «L'Allemagne Fédérale se penche sur son passé ", dans Vingtième Siècle, nº 2. 1985 ; M. Pollack, "La fête d'une autre histoire à Berlin ", dans Vingtième Siècle, 4, 1986, pp. 146-148.

6. P. Ayçoberry, Cologne entre Napoléon et Bismarck. La croissance d'une ville rhénane, Paris, 1981 ; voir aussi son livre sur La Question nazie, Paris, 1979 ; pour un recensement des travaux français sur l'histoire sociale et économique allemande, voir : G. Krumeich, « Französische Arbeiten seit

1945 zur deutschen Sozial - und Wirtschaftsgeschichte im 19. und 20. Jahrhundert », dans Geschichte und Gesellschaft 12. 1986, pp. 106-133 ; voir en outre, N. Pietri, Evolution économique de l'Allemagne du milieu du XIX $X^{\mathrm{e}}$ siècle à 1914, Paris, 1982 ; J. Michel, «Politique syndicale et conjoncture économique : la limitation de la production de charbon chez les mineurs européens au XIX ${ }^{\mathrm{e}}$ siècle », Le mouvement social, $\mathrm{n}^{\circ} 119$, avril-juin 1982 ; id. « L'échec de la grève générale des mineurs européens avant 1914 ", Revue d'histoire moderne et contemporaine, 1982. Quelques chiffres étonnants concernent l'intérêt des Français pour l'histoire allemande : en 1976 l'Allemagne se trouve en tête des pays étrangers traités par des thèses d'État et des thèses de 3e cycle en histoire moderne et contemporaine; $12 \%$ portaient sur l'Allemagne, seulement $8 \%$ sur l'Angleterre, $5 \%$ sur l'Italie, 5 \% sur les États-Unis, $5 \%$ sur l'Union soviétique (calculs effectués sur la base des statistiques publiées dans la Lettre d'information de l'Institut d'Histoire moderne et contemporaine, $\mathrm{n}^{\circ}$

7.1983). 
7. Cette série de rencontres est organisée par un groupe d'initiative qui rassemble Patrick Fridenson, Heinz-Gerhard Haupt, Yves Lequin et moi-même.

\section{AUTEURS}

\section{HARTMUT KAELBLE}

Hartmut Kaelble est professeur à l'Institut d'Histoire économique et sociale de l'Université libre de Berlin Ouest, ZI6, Hittorfstrasse 2-4, 1000 Berlin 33. 